\title{
Photoluminescence of negatively charged excitons in high magnetic fields
}

\author{
M. Hayne \\ Laboratorium voor Vaste-Stoffysica en Magnetisme, Katholieke Universiteit Leuven, Celestijnenlaan 200D, B-3001 Leuven, Belgium \\ and Department of Physics, University of Exeter, Stocker Road, Exeter EX4 4QL, United Kingdom \\ C. L. Jones* \\ Department of Physics, University of Exeter, Stocker Road, Exeter EX4 4QL, United Kingdom \\ R. Bogaerts \\ Laboratorium voor Vaste-Stoffysica en Magnetisme, Katholieke Universiteit Leuven, Celestijnenlaan 200D, B-3001 Leuven, Belgium \\ C. Riva \\ Departement Natuurkunde, Universitaire Instelling Antwerpen, Universiteitsplein 1, B-2610 Antwerpen, Belgium \\ A. Usher \\ Department of Physics, University of Exeter, Stocker Road, Exeter EX4 4QL, United Kingdom \\ F. M. Peeters \\ Departement Natuurkunde, Universitaire Instelling Antwerpen, Universiteitsplein 1, B-2610 Antwerpen, Belgium \\ F. Herlach and V. V. Moshchalkov \\ Laboratorium voor Vaste-Stoffysica en Magnetisme, Katholieke Universiteit Leuven, Celestijnenlaan 200D, B-3001 Leuven, Belgium \\ M. Henini \\ Department of Physics, University of Nottingham, University Park, Nottingham NG7 2RD, United Kingdom
}

(Received 17 March 1998; revised manuscript received 21 September 1998)

\begin{abstract}
We have studied the low-temperature photoluminescence of the two-dimensional electron gas in a single GaAs quantum well in magnetic fields up to $50 \mathrm{~T}$ over four orders of magnitude of illumination intensity. At the very highest illumination powers, where the recombination is excitonic at zero field, we find that the binding energy of both the singlet and triplet states of the negatively charged exciton $\left(X^{-}\right)$increase monotonically with the applied field above $15 \mathrm{~T}$. This contradicts recent calculations for $X^{-}$, but is in agreement with adapted calculations for the binding energy of negative-donor centers. At low-laser powers we observe a strong transfer of luminescence intensity from the singlet (ground) state to the triplet (excited) state as the temperature is reduced below $1 \mathrm{~K}$. This is attributed to the spin polarization of the two-dimensional electron gas by the applied magnetic field. [S0163-1829(99)01104-2]
\end{abstract}

\section{INTRODUCTION}

The optical properties of two-dimensional electron gases (2DEG's) remains a subject of intense interest. Recently, our understanding of photoluminescence (PL) spectra from 2DEG's has been much advanced by the study of charged excitons or trions, ${ }^{1-6}$ which are formed when two electrons bind with a hole (negatively charged excitons $X^{-}$), ${ }^{1-5}$ or when an electron binds with two holes (positively charged excitons $\left.X^{+}\right)^{5,6}$ In undoped quantum wells photoexcited electron-hole pairs form neutral Mott-Wannier excitons $\left(X^{0}\right)$, analogous to the hydrogen atom. Introducing an excess electron (or hole) density results in the formation of $X^{-}$ $\left(X^{+}\right)$, with only the ground-singlet state being occupied in zero-magnetic field. Further increases in the density (to greater than $5 \times 10^{10} \mathrm{~cm}^{-2}$ ) result in the smooth evolution of the singlet recombination at zero-magnetic field into that of free carriers. ${ }^{3}$

The corresponding situation with a magnetic field $B$ ap- plied perpendicular to the plane of the 2DEG is less well understood. For undoped wells the field increases the $X^{0}$ binding energy by reducing the Bohr radius, squeezing the electron and hole closer together. ${ }^{7}$ For dilute 2DEG's the same process also allows observation of the triplet state of the $X^{-}$, which is unbound in zero field. ${ }^{2-5}$ However, due to the fact that $X^{-}$is a many-body system involving the interaction of two electrons with a hole, the physics is considerably more complex than for the $X^{0}$, and a straightforward increase of the binding energy with magnetic field is not always expected. ${ }^{8,9}$

Here, we present the results of PL experiments in highmagnetic fields in which the laser-excitation power is varied by four orders of magnitude. At our highest laser powers the appearance of $X^{0}$ in the spectrum allows us to measure the binding energies of the singlet and triplet states of the $X^{-}$. Our results are in contradiction to a recent theory, ${ }^{8}$ which predicts a decrease of the singlet-binding energy at fields above $10 \mathrm{~T}$, and a triplet ground state above $30 \mathrm{~T}$. In con- 
trast, adapting a theory for the negatively charged donor center ${ }^{9}$ gives good agreement with our experimental data. At lower laser powers, where the observation of recombination from the second Landau level identifies the 2DEG density to be $1.2 \times 10^{11} \mathrm{~cm}^{-2}$, the formation of $X^{-}$splits the lowestLandau level recombination peak above $10 \mathrm{~T}$. Reducing the temperature below $1 \mathrm{~K}$ induces an anomalous transfer of intensity from the low-energy (singlet) peak to the highenergy (triplet) peak. This behavior is explained in terms of the spin polarization of the 2DEG.

\section{EXPERIMENTAL DETAILS}

The sample studied was a $\mathrm{GaAs} / \mathrm{Al}_{x} \mathrm{Ga}_{1-x} \mathrm{As}$ asymmetric modulation-doped single quantum well of width $102 \AA$ grown by molecular-beam epitaxy, with 2 DEG density $\left(n_{s}\right)$ of $3.7 \times 10^{11} \mathrm{~cm}^{-2}$ and mobility of $5 \times 10^{5} \mathrm{~cm}^{2} \mathrm{~V}^{-1} \mathrm{~s}^{-1}$ subsequent to saturation of the persistent photoconductivity effect. ${ }^{10}$ For the results reported here, $n_{s}$ was reduced using above- $\mathrm{Al}_{x} \mathrm{Ga}_{1-x}$ As-band-gap illumination ${ }^{11,12}$ to about 1.2 $\times 10^{11} \mathrm{~cm}^{-2}$. The observation of significant bulk GaAs PL from the substrate, coupled with increased density depletion in comparison to that calculated from theory, ${ }^{11}$ leads us to believe that a less well-known density-depletion effect involving charge separation in the GaAs (Ref. 13) is also at work. However, the details of the density depletion will not be discussed here. The experiments were conducted in $\mathrm{dc}$ magnetic fields to $15 \mathrm{~T}$ at temperatures between $20 \mathrm{mK}$ and $1.2 \mathrm{~K}$ in a dilution refrigerator, and in pulsed magnetic fields up to $50 \mathrm{~T}$ using a bath cryostat at $4.2 \mathrm{~K}$. In both cases the field was applied parallel to the growth $(z)$ direction. For the experiments in dc magnetic field the sample was excited using an argon-ion laser via a single-optical fiber with a core diameter of $125 \mu \mathrm{m}$. The same fiber was used to collect the PL. For the pulsed-field experiments the light from a frequency-doubled solid-state laser (operating at $532 \mathrm{~nm}$ ) was transmitted to the sample down the center of a bundle of $400-\mu \mathrm{m}$ core fibers, with the PL being collected by the fibers at the edge of the bundle. The detector was gated at the top of the 20-ms field pulse, giving a photon integration time of $1.84 \mathrm{~ms}$ with a field resolution of $\pm 1 \%$. The spectral resolution of the experiment was $\leqslant 0.5 \mathrm{meV}$.

\section{EXPERIMENTAL RESULTS}

Figure 1(a) shows the evolution of the PL peaks for data taken in the dilution refrigerator at an incident power density of $1.3 \mathrm{~mW} \mathrm{~cm}^{-2}$ and a bath temperature of $20 \mathrm{mK}$. Similar behavior was observed at laser powers of 0.3, 0.7, 12, and 25 $\mathrm{mW} \mathrm{cm}{ }^{-2}$. Indeed, as can be seen from Fig. 1(b), the data taken in the pulsed-field magnet at the considerably higher laser power of $380 \mathrm{~mW} \mathrm{~cm}^{-2}$ show the same basic features. At low field, the decay of the second Landau level identifies the position of $\nu=2$, giving $n_{s}=1.2 \times 10^{11} \mathrm{~cm}^{-2}\left(n_{s}\right.$ $=e B \nu / h)$ and at high field a new line $(T)$ emerges on the high-energy side of the lowest energy recombination $(S)$. The shift of the higher power data to higher energies (over and above that introduced in Fig. 1 for clarity) may be attributed to the reduction in the band bending due to the increased illumination intensity. ${ }^{3}$ A second possible mechanism is band-gap renormalization, ${ }^{3,14}$ but since the density is the

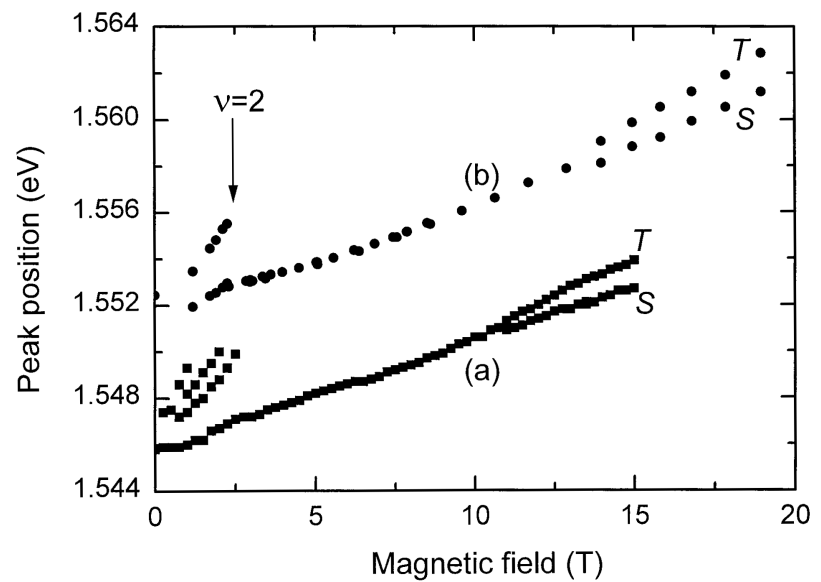

FIG. 1. Dependence of the peak energy on magnetic field at a power of (a) $1.3 \mathrm{~mW} \mathrm{~cm}^{-2}$ and (b) $380 \mathrm{~mW} \mathrm{~cm}^{-2}$. In the former case the data were taken at a bath temperature of $20 \mathrm{mK}$ in a superconducting magnet, and in the latter case at $4.2 \mathrm{~K}$ in pulsed fields. The data shown in (b) have been shifted up in energy by 4 meV for clarity.

same in both cases, this is not expected to make a significant contribution. Note that in both sets of data in Fig. 1 the splitting between $S$ and $T$ is the same size at a given field, but is resolved to lower fields in the low-temperature data taken in the dc magnet. The emergence of the new line $T$ for the data of Fig. 1(a) between 10 and $14 \mathrm{~T}$ is shown in Fig. 2. It can be seen that $T$ rapidly increases in intensity, at the expense of $S$, completely dominating the spectrum by $14 \mathrm{~T}$. Despite the fact that $T$ is at higher energy than $S$, we find that warming the sample reduces its relative intensity. The inset to Fig. 2 shows data taken under the same conditions as the main part of the figure, but with a bath temperature of $1.2 \mathrm{~K}$. In contrast to the low-temperature case, $S$ is not so readily suppressed at high-magnetic fields, and the two peaks are of similar intensity at $15 \mathrm{~T}$.

Despite the similarity of the PL over three orders of magnitude of laser power, a further increase in laser power by a

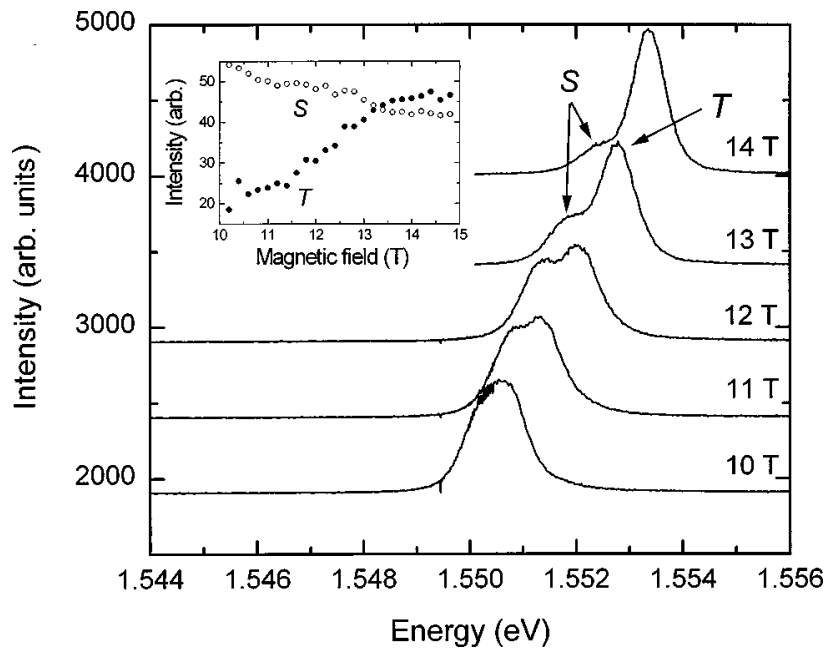

FIG. 2. PL spectra for the low-temperature low-power data of Fig. 1(a). The curves have been offset in intensity for clarity. The inset shows the peak intensities at $1.2 \mathrm{~K}$ over the same field range and for the same laser power. 


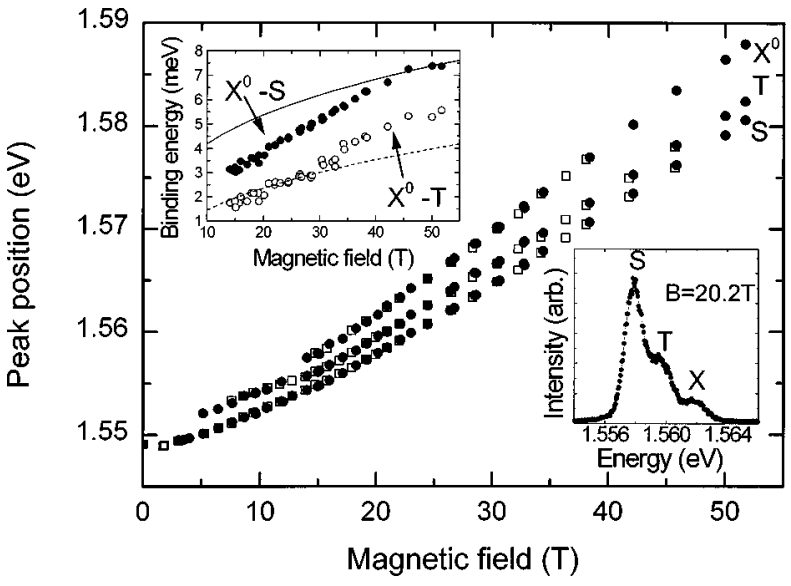

FIG. 3. Dependence of the peak energy on magnetic field at a power of $1300 \mathrm{~mW} \mathrm{~cm}^{-2}$ (squares). Also shown are data taken at $3800 \mathrm{~mW} \mathrm{~cm}^{-2}$ (circles). The bath temperature is $4.2 \mathrm{~K}$. The upper inset shows the experimental (theoretical) binding energy of the $X^{-}$ states, [singlet, closed symbols (full line); triplet, open symbols (dashed line)], while the lower inset shows a PL spectrum at 20.2 T.

factor of 3 produces a qualitative change in the data. Figure 3 shows the energy dependence of the luminescence peaks taken at laser powers of 1300 and $3800 \mathrm{~mW} \mathrm{~cm}^{-2}$ (corresponding to incident powers of 6.8 and $20 \mathrm{~mW}$, respectively) in the pulsed magnet at a bath temperature of $4.2 \mathrm{~K}$. Comparison of the data of Fig. 1(b) up to $50 \mathrm{~T}$ (not shown) with that of Fig. 3, shows that the lines $S$ and $T$ are the same features in both cases. However, there are two significant differences between the two figures. The first is that we no longer observe recombination from electrons in the second Landau level. Second, a new, but considerably less intense line has appeared to even higher energy than $T$. We also note that the increase of laser power from 1300 to $3800 \mathrm{~mW} \mathrm{~cm}^{-2}$ has no effect on the positions of the recombination lines (Fig. 3 ), but increases the relative intensity of the new line by a factor of 1.5. Before discussing the reasons for these differences, we shall use the data of Fig. 3 to identify the lines we have observed.

We attribute the highest-energy line in Fig. 3 to recombination of the neutral exciton, and $S$ and $T$ to recombination of the singlet and triplet states of the negatively charged exciton, respectively, both corresponding to a change in the total $z$ component of the spin of +1 . Compelling evidence in favor of these assignments is given by comparing our data with that of Shields et al.; the data of Fig. 3 bear a striking resemblance to that of Fig. 1(b) of Ref. 2, with the exception that we cannot identify recombination from the singlet state with a change in the total $z$ component of the spin of -1 . At low field this difference may be attributed to the width of our luminescence lines, and at high-magnetic fields to the fact that the Zeeman splitting raises the energy of this level above that of the observed-triplet state. Further evidence in favor of this assignment is the relative increase in the strength of the $X^{0}$ peak upon increasing the laser power, which is to be expected for three reasons. In the first instance, the density of photoexcited holes is proportional to the laser power, thus increasing the laser power will increase the relative density of holes in the quantum well, favoring the formation of $X^{0}$. Second, although $n_{s}$ is constant from 0.3 to $380 \mathrm{~mW} \mathrm{~cm}^{-2}$, a further decrease cannot be ruled out. This would have a similar effect as increasing the number of holes. Finally, significant sample heating could evaporate the excess electron from the $X^{-}$, leaving $X^{0}$ and a free electron. Although sample heating could not result in the disappearance of the second Landau level, the other mechanisms might also be invoked to explain the transition from the behavior of Fig. 1 to that of Fig. 3. Using a value for the recombination time in the quantum well of $1 \mathrm{~ns}$, the hole density in the quantum well is estimated to be $3 \times 10^{9} \mathrm{~cm}^{-2}$ at our highest-laser power. Thus, the hole density is beginning to become comparable to $n_{s}$, particularly if the latter has decreased further. However, the dominant mechanism behind the marked change in the data of Figs. 1 and 3 is not fully understood at present.

\section{DISCUSSION}

In the previous section we presented our experimental results, and identified the recombination lines $S$ and $T$ as that of the singlet and triplet states of the $X^{-}$. Here we discuss some rather unexpected features of the $X^{-}$PL, which warrant explanation. Shields et al. have conducted experiments on $X^{-}$in a $300-\AA$ GaAs quantum well to fields of $20 \mathrm{~T}^{4}{ }^{4}$ They find that the binding energy of both the triplet and singlet states, as measured by the separation from the $X^{0}$ line, increases rapidly at fields up to $10 \mathrm{~T}$, and then saturates. Calculations of the triplet-state binding energy by Whittaker and Shields ${ }^{8}$ were found to be in good agreement with this experimental data, however, in the case of the singlet the agreement was rather poor. The same authors also predicted a decrease in the singlet-binding energy above $10 \mathrm{~T}$ for $X^{-}$ in a $100-\AA ̊$ quantum well, leading to a cross over between the singlet and triplet states at $30 \mathrm{~T}^{8}$ The upper inset to Fig. 3 shows the binding energies of the singlet and triplet states as measured from our experimental data, for fields from 15 to $50 \mathrm{~T}$. Due to the broad luminescence lines in our data, the peaks cannot be properly distinguished at lower field, however, it is quite clear that at high fields the binding energies of both states increase linearly in magnetic field with a slightly larger slope for the singlet state. The monotonic increase of binding energy with magnetic field is in contrast to the experimental data of Shields et al. ${ }^{4}$ which we believe is a consequence of the reduction in well width. However, it is interesting that both sets of data show an approximately constant separation between the singlet and triplet states. More significant is that our results are in direct contradiction with the qualitative prediction of the theory for a $100-\AA$ quantum well, as well as giving values that are about a factor of 2 larger. ${ }^{8}$

Such a large discrepancy between theory and experiment warrants further investigation. To this end we have adapted recent calculations ${ }^{9}$ for negative donor centers $\left(D^{-}\right)$with a magnetic field applied in the $z$ direction. These are formed when a neutral shallow donor binds an extra electron, and are thus equivalent to $X^{-}$in the case that the hole has an infinite mass. For a finite-mass hole, we calculate the $X^{-}$energy by replacing the in-plane mass of the electron, $\mathrm{m}^{*} / \mathrm{m}_{0}=0.068$, in the $D^{-}$problem by the one of a particle with the excitonic-reduced mass, i.e., $\mu / m_{0}=0.04$. Following Riva, Peeters, and Schweigert ${ }^{9}$ we define the binding energy of the excess electron, 


$$
E_{b}^{n}\left(X^{-}, L\right)=E^{0}\left(X^{0}, 0\right)+E(e, 0)-E^{n}\left(X^{-}, L\right),
$$

where $E^{0}\left(X^{0}, 0\right)$ is the binding energy of the neutral exciton, $E(e, 0)$ is the energy of an electron in the lowest Landau level, and $E^{n}(X, L)$ is the $n$th energy level of the $X^{-}$with orbital-angular momentum $L$ in the $z$ direction. As shown in the upper inset of Fig. 3, the results of this calculation are in remarkable agreement with the experimental results, even though the theoretical results should only be taken as a rough estimate for the $X^{-}$binding energies. Not only does the theory reproduce the monotonic increase of the binding energy with the magnetic field, but it lies reasonably close to the experimental data. At present we have no explanation as to why our experimental and theoretical results should be in such broad disagreement with the theory of Whittaker and Shields. Comparison with the negatively charged donor case would lead us to expect more exotic behavior of the binding energy to occur as the electrons and holes become separated. This would be more easily achieved in a wide-quantum well than a narrow one. In contrast, the theory of Whittaker and Shields predicts the opposite behavior. More work on this problem is clearly required.

In Fig. 2 we demonstrated the anomalous temperature dependence of the $S$ and $T$ lines below $1 \mathrm{~K}$. Identifying these lines as the singlet and triplet states of the negatively charged exciton does not solve this anomaly, since it is the intensity of the ground (singlet) state that is suppressed at low temperature. One possible explanation for this behavior can be found by examining the electron-spin polarizations of the singlet and triplet states of $X^{-}$[see, for example, Fig. 1(c) of Ref. 2]. The singlet state involves the antisymmetric combination of the spin of the electrons with two possible alignments of the hole spin. In contrast, the two lower states of the triplet involve symmetric-field aligned electron spins and antisymmetric-spatial wave functions. For the high-magnetic fields and very low temperatures $(20 \mathrm{mK})$ of Fig. 1(a) the 2DEG will be totally spin polarized, thus only spin-triplet states will form. Singlet states will subsequently form via the relaxation of one of the spins of the electrons, unless the spin-relaxation time is longer than the recombination time. This is the case for free electrons in quantum wells, ${ }^{15}$ and might also be the case for $X^{-}$, where the electrons are weakly bound. Increasing the temperature has the effect of depolarizing the $2 \mathrm{DEG}$, and results in the reappearance of the singlet state. Taking the magnitude of the electronic $g$ factor for a $100-\AA$ quantum well ${ }^{16}$ to be 0.2 we find that the Zeeman energy is $2 \mathrm{~K}$ at $15 \mathrm{~T}$, which supports our explanation. However, even at the highest fields in the pulsed magnet the singlet state remains the strongest feature, despite the fact that our estimate for the Zeeman splitting gives $6 \mathrm{~K}$ at $45 \mathrm{~T}$. There are a number of possible explanations for this; for example, that our estimate of the $g$ factor is an upper limit, or that in the pulsed-field experiment there is significant electron heating due to the high-laser power. Alternatively, it could be that as the magnetic field increases the binding energy, the electron spin-relaxation rate also increases, becoming less like that of free electrons, and more like that of neutral excitons. ${ }^{15}$ This process might also be enhanced by the reduction in the electron-hole separation, which results from the flattening of the bands caused by the illumination.

Before concluding we would like to point out that very similar behavior was first observed by Heiman et al. ${ }^{17}$ in multiple quantum-well samples, who attributed it to the fractional quantum-Hall effect. This conclusion was reached because the high-energy PL peak appeared at a Landau-level filling factor $\nu$ of about $2 / 3$, and because the temperature dependence implied a gap that was much smaller than the splitting between the peaks, but of similar size to the minima at $\nu=2 / 3$ in the longitudinal resistivity. A number of groups subsequently reported features in the PL such as shifts ${ }^{18}$ and intensity oscillations ${ }^{19}$ in a variety of structures, all of which were given a similar interpretation. We do not suggest that all of these interpretations were incorrect, but it is certainly the case that the identification of $X^{-}$has considerably improved our understanding of the PL of 2DEG's in highmagnetic fields. Indeed, recent experiments ${ }^{20}$ on single $\mathrm{GaAs} / \mathrm{Al}_{x} \mathrm{Ga}_{1-x} \mathrm{As}$ heterojunctions to filling factors as low as $1 / 35$ show no PL features that can be attributed to the Wigner crystal, ${ }^{21}$ but the results are also explained in terms of negatively charged excitons.

\section{CONCLUSIONS}

We have studied the photoluminescence of the 2DEG in a single narrow GaAs quantum well in high magnetic fields. The recently predicted crossover of the binding energies of the singlet and triplet states of the $X^{-}$at $30 \mathrm{~T}$ is not observed, rather the binding energies increase monotonically with field above $15 \mathrm{~T}$. This behavior is reproduced by adapting theory developed for the study of negatively charged donor centers. The anomalous transfer of intensity from the singlet to the triplet state as the temperature is reduced below $1 \mathrm{~K}$ in high fields is attributed to spin polarization of the 2DEG.

\section{ACKNOWLEDGMENTS}

This work was supported by the EPSRC (UK), the FWOVlaanderen, the Flemish GOA, and the Belgian IUAP programs. M. Hayne and R.B. acknowledge the support of the FWO-Vlaanderen. The authors would like to thank A. J. Shields for helpful discussions.
*Present address: Wolfson College, Oxford, U.K.

${ }^{1}$ K. Keng, R. T. Cox, Y. Merle d'Auibigne, F. Bassani, K. Saminadar, and S. Tatarenko, Phys. Rev. Lett. 71, 1752 (1993).

${ }^{2}$ A. J. Shields, M. Pepper, M. Y. Simmons, and D. A. Ritchie, Phys. Rev. B 52, 7841 (1995).

${ }^{3}$ A. J. Shields, M. Pepper, D. A. Ritchie, and M. Y. Simmons, Adv. Phys. 44, 47 (1995).

${ }^{4}$ A. J. Shields, M. Pepper, P. C. M. Christiansen, J. C. Maan, M.
Y. Simmons, and D. A. Ritchie, in High Magnetic Fields in the Physics of Semiconductors, edited by G. Landwehr and W. Ossau (World Scientific, Singapore, 1997), Vol. II, p. 737.

${ }^{5}$ G. Finkelstein, H. Shtrikman, and I. Bar-Joseph, Phys. Rev. B 53, R1709 (1996); D. Gekhtman, E. Cohen, A. Ron, and L. N. Pfeiffer, ibid. 54, 10320 (1996).

${ }^{6}$ A. J. Shields, J. L. Osborne, M. Y. Simmons, M. Pepper, and D. A. Ritchie, Phys. Rev. B 52, R5523 (1995); Y. V. Ponomarev, 
A. Usher, P. J. Rodgers, B. L. Gallagher, M. Henini, and G. Hill, ibid. 54, 13891 (1996).

${ }^{7}$ D. M. Whittaker and R. J. Elliot, Solid State Commun. 68, 1 (1988)

${ }^{8}$ D. M. Whittaker and A. J. Shields, Phys. Rev. B 56, 15185 (1997).

${ }^{9}$ C. Riva, F. M. Peeters, and V. A. Schweigert, Phys. Rev. B 57, 15392 (1998).

${ }^{10}$ C. R. H. White, M. Davies, M. Henini, B. R. Davidson, P. C. Main, J. R. Owers-Bradley, L. Eaves, O. H. Hughes, M. Heath, and M. S. Skolnick, Semicond. Sci. Technol. 5, 792 (1990).

${ }^{11}$ M. Hayne, A. Usher, A. S. Plaut, and K. Ploog, Phys. Rev. B 50, 17208 (1994).

${ }^{12}$ A. S. Chaves, A. F. S. Penna, J. M. Worlock, G. Weimann, and W. Schlapp, Surf. Sci. 170, 168 (1986); D. Richards, G. Fasol, and K. Ploog, Appl. Phys. Lett. 57, 1099 (1990).

${ }^{13}$ A. J. Shields, J. L. Osborne, M. Y. Simmons, D. A. Ritchie, and M. Pepper, Semicond. Sci. Technol. 11, 890 (1996).

${ }^{14}$ C. Delalande, J. Orgonasi, M. H. Meynadier, J. A. Brum, G.
Bastard, G. Weimann, and W. Schlapp, Solid State Commun. 59, 613 (1986).

${ }^{15}$ R. T. Harley and M. J. Snelling, Phys. Rev. B 53, 9561 (1996).

${ }^{16}$ M. J. Snelling, G. P. Flinn, A. S. Plaut, R. T. Harley, A. C. Tropper, R. Eccleston, and C. C. Phillips, Phys. Rev. B 44, 11345 (1991).

${ }^{17}$ D. Heiman, B. B. Goldberg, A. Pinczuk, C. W. Tu, A. C. Gossard, and J. H. English, Phys. Rev. Lett. 61, 605 (1988).

${ }^{18}$ B. B. Goldberg, D. Heiman, A. Pinczuk, L. Pfeiffer, and K. West, Phys. Rev. Lett. 65, 641 (1990); H. Buhmann, W. Joss, K. von Klitzing, I. V. Kukushkin, G. Martinez, A. S. Plaut, K. Ploog, and V. B. Timofeev, ibid. 65, 1056 (1990).

${ }^{19}$ A. J. Tuberfield, S. R. Haynes, P. A. Wright, R. A. Ford, R. G. Clark, J. F. Ryan, J. J. Harris, and C. T. Foxon, Phys. Rev. Lett. 65, 637 (1990).

${ }^{20}$ A. N. Priest, R. J. Nicholas, H. H. Cheng, M. van der Burgt, J. J. Harris, and C. T. Foxon, Physica B 249-251, 562 (1998).

${ }^{21}$ E. M. Goldys, S. A. Brown, R. B. Dunford, A. G. Davies, R. Newbury, R. G. Clark, P. E. Simmonds, J. J. Harris, and C. T. Foxon, Phys. Rev. B 46, 7957 (1992); and references therein. 